\title{
Vitesses et temps ferroviaires
}

Un chapitre du programme scientifique de l'AHICF, 2008-2013

François Caron et Etienne Auphan

\section{CpenEdition}

\section{Journals}

Édition électronique

URL : https://journals.openedition.org/rhcf/861

DOI : 10.4000/rhcf.861

\section{Éditeur}

Rails \& histoire

Édition imprimée

Date de publication : 1 juin 2008

Pagination : 103-109

ISSN : 0996-9403

\section{Référence électronique}

François Caron et Etienne Auphan, « Vitesses et temps ferroviaires », Revue d'histoire des chemins de fer [En ligne], 39 | 2008, mis en ligne le 01 juin 2011, consulté le 22 avril 2022. URL : http:// journals.openedition.org/rhcf/861 ; DOI : https://doi.org/10.4000/rhcf.861 
François CARON et Etienne AUPHAN

\section{Vitesses et temps ferroviaires}

Un chapitre du programme scientifique de I'AHICF, 2008-2013*

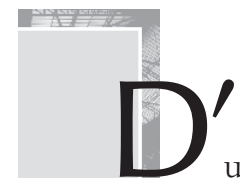

une façon générale, la vitesse est la mesure de la distance parcourue dans un temps donné. Le chemin de fer a fait franchir un saut à la vitesse ferroviaire, au moins pendant près d'un siècle. Dans les premiers temps du développement du chemin de fer même, la vitesse des convois et la contraction du temps des voyages et des transports de marchandises furent perçues comme leurs principales nouveautés. Mais le temps ferroviaire a très tôt été découpé en catégories, comme celles de la petite et de la grande vitesse pour les marchandises. Dès lors le train est souvent apparu comme un symbole de lenteur et non de vitesse, plus encore au fur et à mesure du remplacement du réseau routier issu du XIX ${ }^{\mathrm{e}}$ siècle par le nouveau réseau rapide adapté à l'automobile. L'histoire de la vitesse ferroviaire ne peut donc se réduire à celle des records et des performances réalisées par les "rapides et express » sur les grands axes du réseau ni à celle des impressions éprouvées par les voyageurs des trains rapides lorsqu'ils regardent par la fenêtre des voitures. Elle doit être

\footnotetext{
* Ce texte adopté par le Comité scientifique de l'AHICF en mai 2008 oriente désormais une partie des recherches de l'association appuyées sur une journée scientifique en 2010 et un colloque en 2011, année qui verra le $30^{\mathrm{e}}$ anniversaire de l'ouverture de la première ligne à grande vitesse en France.
} 
replacée dans son contexte technique, socioéconomique et socioculturel. En d'autres termes : la vitesse, pour qui, pour quoi ? Que faire du temps gagné par la vitesse (pour un voyageur) et que faire de la marchandise expédiée ou reçue plus tôt (pour l'utilisateur) ? Derrière ces interrogations se profile la valeur économique du temps : quel est le prix que l'usager du transport est prêt à payer pour que son transport soit plus rapide ou pour recevoir plus rapidement ce qu'il attend (demande) ? Ou, formulée de manière inverse, du côté de l'offre : quel est le coût que l'exploitant est prêt à assumer pour mieux répondre à la demande de rapidité ? La réponse est naturellement multiforme en fonction de la nature de la clientèle et des types de transport, mais aussi des conditions du marché de l'époque. Elle doit prendre en compte la vitesse de circulation des marchandises aussi bien que celle des voyageurs.

Pour réaliser un tel programme, il semble pertinent :

1- d'analyser dans un premier temps la perception que les exploitants ont eue de la vitesse et des besoins des usagers (ou clients) dans ce domaine ;

2 - dans un deuxième temps, l'influence que les politiques de vitesses ont eue sur l'évolution des techniques ferroviaires ;

3- dans un troisième temps, les transformations du temps ferroviaire du point de vue des vitesses en ligne, des temps de parcours ou des horaires, et les modalités de l'adaptation de l'exploitation et du comportement des voyageurs à ces contraintes temporelles.

\section{La perception des besoins : les fondements des politiques de vitesse}

Il faut s'interroger sur les raisons qui ont justifié l'adoption des politiques de vitesse et définir les systèmes de représentation qui expliquent les orientations prises par ces politiques. L'hypothèse de départ est qu'il faut prendre en compte de manière prioritaire dans une telle analyse la vision que les exploitants ont pu avoir des attentes et des besoins des utilisateurs du réseau. Cette vision peut être inspirée aussi bien par des préjugés et des a priori sociaux et culturels que par les leçons tirées de l'expérience ou par des formalisations à prétention scientifique. Un dialogue plus ou moins confus s'établit entre les voyageurs et les expéditeurs d'un côté, les ingénieurs chargés de concevoir les services de l'autre. Ce dialogue s'est peu à peu organisé et institutionnalisé. À partir de l'entre-deux-guerres et, surtout, des années 1950, il a reposé sur des enquêtes d'opinion, dont il conviendrait d'écrire l'histoire. Elles jouèrent à la SNCF un rôle considérable dans les prises de décision. Les ingénieurs y trouvaient un moyen de satisfaire leur besoin de rigueur et de rationalité. 
On peut s'interroger sur les origines de l'invention du voyageur pressé et plus généralement de "l'homme pressé », tel que décrit par Paul Morand. Il a joué un rôle considérable dans le système de représentation des exploitants. On doit s'interroger aussi sur la différenciation des classes en fonction de la vitesse tout autant qu'en fonction du confort. Une attention toute particulière doit être portée à l'étude des conceptions des exploitants des compagnies et de la SNCF dans le domaine des « rapides et express » et au changement qui s'est produit dans ce domaine dans le courant des années 1960. Ainsi, selon la période envisagée, il conviendra de retenir tel ou tel aspect de la vitesse ferroviaire, notamment :

- la vitesse comme argument publicitaire. Rappelons-nous quelques slogans caractéristiques d'une époque : « Paris - Lyon en 4 h 15 », « 100 villes à plus de 100 », « Gagner du temps sur le temps... »;

- la vitesse (positive ou négative) indirectement valorisée sous la forme de l'utilisation de la durée du parcours : «le temps d'un déjeuner, vous ferez Paris - Rennes » ou bien « le temps d'une vraie nuit entre Paris et Toulouse »...

Des analyses du même ordre doivent être appliquées aux transports de marchandises et tout particulièrement à celle des transports dits de grande vitesse ou encore du « régime accéléré » et du « régime ordinaire » à la SNCF. Il faudrait, dans une telle perspective, porter une attention particulière à la vitesse comme argument commercial du transport de fret, par exemple pour le transport des animaux et des denrées périssables ou pour la messagerie. On ne peut qu'être étonné de la lenteur avec laquelle les mentalités ont évolué dans ce domaine. D’une manière générale, le rôle de cette variable a été très largement sous-estimé dans l'histoire des politiques de transports de marchandises, dont la caractéristique principale fut longtemps la lenteur...

Les sources pour écrire une telle histoire ne manquent pas : traités et manuels, articles, archives des compagnies, enquêtes d'opinion et analyses sociologiques sur les budgets temps, publicité ferroviaire.

\section{Vitesse, temps de parcours et techniques ferroviaires}

C'est une banalité de dire que l'histoire de l'élaboration et de la réalisation des politiques de vitesses doit être replacée dans le cadre du système ferroviaire global. Il conviendra donc de porter une attention diachronique sur les « coulisses » techniques de la vitesse : investissements concernant l'infrastructure (suppression des limitations ponctuelles de vitesse par simplification du plan de voie, rectification des courbes, amélioration de l'alimentation électrique et adaptation de la signalisation, suppression des passages à niveaux...) et le matériel roulant (dispositifs de freinage, bogies...) grâce auxquels s'élabore 
tout particulièrement la vitesse en ligne autour de deux pôles bien distincts : celui de la réalisation de records et de performances extrêmes, celui de la limitation réglementaire des vitesses.

Les techniques de la traction du matériel roulant et des installations fixes doivent maintenir entre elles une symbiose parfaite. Leur histoire doit donc s'écrire dans la perspective d'une analyse de leurs interférences. Charles Boyaux, dans un rapport présenté au conseil d'administration de la SNCF en 1958, indiquait que la vitesse limite des trains de voyageurs n'était qu'un élément d'une évolution d'ensemble dont les paramètres étaient interdépendants. Il ajoutait que "la possibilité de circuler à $140 \mathrm{~km} /$ heure n'était qu'un sousproduit de la puissance installée ». En réalité les réseaux ont utilisé très précocement les travaux d'entretien et de renouvellement des voies et des établissements pour introduire des aménagements qui permettaient d'accrồtre les vitesses sur les axes principaux. Un programme dit « d'équipement des voies en vue de permettre des circulations en grande vitesse » fut défini et entrepris dans l'entre-deux-guerres et poursuivi par la SNCF. Sa réalisation, selon un rapport de 1967, a d'ailleurs été grandement favorisée par le fait que « le tracé des lignes retenu à l'origine contenait en puissance des possibilités d'accélération ». Les interdépendances à l'intérieur du système ne concernent pas seulement la circulation des trains. Elles intéressent aussi l'aménagement des gares et leur accessibilité, la régulation des trains de marchandises ou le triage des wagons.

L'attention des historiens s'est concentrée principalement sur la relation entre vitesse et sécurité. Mais il faut noter que la vitesse peut être un moyen d'assurer la sécurité des flux. C'est ainsi que la possibilité de franchir rapidement les points singuliers est un facteur majeur de sécurité. De plus la vitesse est, dans de très nombreux cas, le seul moyen d'apporter une réponse aux problèmes d'encombrements en l'absence de toute possibilité d'accroissement des infrastructures. Le rapport de Charles Boyaux, que nous venons de citer, avait pour but de démontrer l'absurdité de la demande présentée par le gouvernement, dans le cadre d'un programme d'économies pour le budget d'exploitation de 1958, de réduire à $120 \mathrm{~km} / \mathrm{h}$ les vitesses limites sur les lignes où les $140 \mathrm{~km} / \mathrm{h}$ avaient été autorisés antérieurement. Boyaux montrait, chiffres à l'appui, que la vitesse était au contraire une source importante d'économie en tenant compte aussi bien des installations fixes que du matériel roulant sur les trajets de Paris à Lyon comme de Paris à Bordeaux. Charles Boyaux terminait son rapport par ces mots : «Toutes ces considérations n'ont d'ailleurs de valeur que parce qu'il s'agit de grandes lignes ayant de bons profils et un trafic très dense. Il ne faudrait pas, bien entendu, les extrapoler à des lignes difficiles 
ou à trafic médiocre sur lesquelles la recherche de la grande vitesse serait une erreur coûteuse. » Cette phrase illustre parfaitement la totale dépendance des politiques de vitesse à l'égard des installations fixes. Une histoire du TGV en apporterait une autre illustration. Enfin une histoire exhaustive des relations, parfois tendues, entre les services des installations fixes et les services du matériel et traction réserverait bien des surprises. Mais la relation entre vitesse et matériel roulant est tout aussi fondamentale que la relation entre vitesse et installations fixes. Nous n'insisterons pas sur ce point.

\section{La construction du temps ferroviaire et ses conséquences économiques, sociologiques et culturelles}

- D’une manière générale la régulation de la vitesse est destinée à permettre au voyageur ou à l'expéditeur d'aménager le temps de manière optimale. La variable stratégique devient ici la vitesse commerciale et la durée du parcours. L'histoire des vitesses ferroviaires a de ce point de vue deux facettes : celle de la réduction des temps de parcours sur les grands axes du réseau et les grandes distances, mais aussi celle du « raccourcissement du délai de transport considéré moins comme un supplément de confort que comme une facilité de circulation accessible à tous ", selon l'expression utilisée dès 1946 par le président de la SNCF dans une réunion du conseil. Car l'homme d'affaires et l'homme pressé en général, qu'il soit touriste, fonctionnaire, journaliste, universitaire ou espion n'ont pas été les seules cibles des exploitants, même si les trains spéciaux qui leurs étaient destinés ont eu la vie dure. Pour l'homme d'affaires la cible majeure était la possibilité de régler ses affaires en une seule journée grâce à un aller-retour possible vers la capitale, la métropole régionale ou le chef-lieu de département dans cette seule journée, ce qui devint réalité avec les « trains d'affaires » à partir des années 1960.

- La recherche de l'accroissement de la vitesse commerciale a eu des conséquences importantes sur le fonctionnement du réseau. L'augmentation de la vitesse commerciale (affichée pour le public et perçue par le voyageur sous la forme de la réduction de la durée du parcours) s'est opérée principalement (en dehors de l'augmentation de la vitesse en ligne) par la réduction du nombre des arrêts intermédiaires et le raccourcissement de leur durée. Ce sont ces deux facteurs qui - avant le TGV - ont sans doute eu les effets les plus prégnants sur l'évolution des voyages en train et sur les rapports entre le territoire et le réseau ferré. En effet, la recherche d'arrêts plus brefs a entraîné celle de la suppression des services annexes, soit déjà permise par l'évolution technique de la traction (suppression des échanges de machines), soit spécifiquement 
voulue dans ce but (suppression du services des bagages, de la Poste, de la restauration à quai, de la recomposition des trains à tranches multiples, etc.). Mais dès avant le TGV la vitesse s'est démocratisée. Ce mouvement a pris son essor à partir des années 1880. Il faut en reconstituer avec précision les différentes étapes. L'aboutissement fut le turbotrain qui transforma totalement la desserte des liaisons transversales puis le TGV qui, de ce point de vue, ne fut pas une rupture mais un accomplissement.

Il serait souhaitable que soient entreprises des recherches plus systématiques réalisées soit sur un plan local et ligne par ligne, soit sur un plan général, concernant les différentes variables de construction du temps ferroviaire et de leur combinaison en fonction de la nature des différents trains, qu'il s'agisse des arrêts, des correspondances, des buffets, des horaires ou des cadencements ou encore des relations entre la gare et la ville. Une attention particulière pourrait être portée à des problèmes spécifiques tels que la traversée de Paris, les horaires des trains de nuit, l'accessibilité des gares et la construction des parkings, ou encore le «temps perdu » dans les gares, qui peut être, on le sait, un temps de méditation et forme le complément du temps de l'homme pressé.

- La vitesse est un instrument (à double tranchant) de l'aménagement du territoire. Un rapport de 1969 définissant la politique d'investissement de la SNCF pour l'année suivante parle de «l'amélioration des relations rapides entre les grands centres » pour ce qui concerne les voyageurs (en réalité, il ne s'agissait que des grands centres situés sur un même axe) et de «l'aménagement des transports entre grands centres ». Une importante littérature a été écrite sur chacun de ces deux thèmes. Elle nous semble pourtant encore insuffisante tant du côté des stratégies d'offre que de leur réception par les acteurs locaux et régionaux et de leurs effets sur ces territoires. On a beaucoup parlé de l'effet du TGV sur la métropolisation du territoire. Il faudrait aussi s'interroger avec plus de précision sur les périodes antérieures.

Ainsi, la suppression de nombre d'arrêts intermédiaires a entrainé celle des dessertes rurales les moins fréquentées sur les lignes secondaires, mais aussi sur les grandes lignes parcourues par les trains à grand parcours; elle s'est traduite par la spécialisation des grands express à la desserte des gares de premier niveau (de plus en plus limitées à une aire déterminée), impliquant des correspondances par « omnibus » (puis TER) « de cabotage » vers les gares de niveau inférieur sur la même ligne, traversées sans arrêt par le train concerné. Il en est d'ailleurs de même de la suppression des rebroussements par utilisation de raccordements existants, remis en service ou construits tout exprès, etc. Cette politique a eu pour effet de reporter souvent sur la route les liaisons entre villes 
de second niveau sur un même axe principal (par exemple d'Amboise à Libourne par correspondance à Saint-Pierre-des-Corps et à Angoulême - ou pire, à Bordeaux !).

Naturellement, le TGV a amplifié ces effets qui ont également un aspect tarifaire : la «déterritorialisation » du déplacement introduite par le TGV et l'abandon partiel de la tarification kilométrique ont concrétisé le passage de la distance-temps à la distance-coût. Cette évolution est en réalité l'aboutissement d'une politique tarifaire de la vitesse, d'abord conçue dans l'après-guerre sous la forme d'un supplément limitatif de fréquentation ( $1^{\text {re }}$ classe). À vrai dire, au cours de cette période, c'est surtout la vitesse commerciale de la liaison assurée uniquement en $1^{\text {re }}$ classe qui est « rapide » par comparaison aux liaisons « toutes classes ». Ce n'est qu’à partir de la fin des années 1960 que les investissements réalisés sur l'infrastructure et le matériel permettront d'augmenter notablement la vitesse en ligne, justifiant ainsi un supplément spécifique, jusqu'à ce que la vitesse soit considérée comme « démocratiquement accessible à tous » et que le supplément se fonde progressivement dans une politique de modulation tarifaire pour régulation du trafic dans le temps (tarif tricolore).

De même l'histoire des transports de marchandises à grande vitesse et du régime accéléré de la SNCF mériterait une relecture complète.

Il faut enfin poursuivre les travaux sur les conséquences sociologiques et psychosociologiques de la vitesse et du raccourcissement des parcours en les appuyant sur des enquêtes réalisées auprès des voyageurs pour apporter un complément aux impressions de voyage qui constituent encore aujourd'hui la source principale de ces recherches. 
